\title{
Aerothermodynamic Analysis of Stardust Sample Return Capsule with Coupled Radiation and Ablation \\ R. N. Gupta
}

Reprinted from

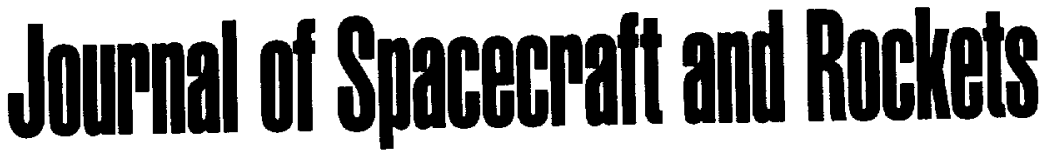

Volume 37, Number 4, Pages 507-514

\section{OALAA}

A publication of the

American Institute of Aeronautics and Astronautics, Inc.

1801 Alexander Bell Drive, Suite 500

Reston, VA 20191-4344 


\title{
Aerothermodynamic Analysis of Stardust Sample Return Capsule with Coupled Radiation and Ablation
}

\author{
Roop N. Gupta* \\ NASA Langley Research Center, Hampton, Virginia 23681
}

\begin{abstract}
An aerothermodynamic analysis of the forebody aeroshell of the Stardust Sample Return Capsule is carried out by using the axisymmetric viscous shock-layer equations with and without fully coupled radiation and ablation. Formulation of the viscous shock-layer equations with shoulder radius as the length scale and implementation of the Vigneron pressure condition allow resolution of the flowfield over the shoulder. With a predominantly supersonic outflow over the shoulder, a globally iterated solution of viscous shock-layer equations can be obtained. The stagnation-point results are obtained along a specified trajectory, whereas detailed calculations along the body are provided at the peak-heating point. The equilibrium calculations with ablation injection are the focus of the present study because of the lack of a general chemical nonequilibrium analysis that accounts for both surface and flowfield effect. The equilibrium calculations also provide a simple way to conserve surface (and flowfield) elemental composition for the current small ablation injection rates, where the surface elemental composition is a mixture of freestream and ablator elements. Therefore, the coupled laminar and turbulent flow solutions with radiation and ablation are obtained by using the equilibrium flow chemistry, whereas a nonequilibrium chemistry model is used for solutions without ablation and turbulence. Various computed results are compared with those obtained by the other researchers.
\end{abstract}

$C_{i}$
$\tilde{C}_{k}$
$C$
$D$
$h_{a}$
$h_{i}$
$\bar{h}_{i}$
$K$
$\bar{K}$
$L$
$\dot{m}$
$n$
$\bar{n}$
$n_{s}$
$P$
$p$
$q$
$\bar{q}$
$q$
$q$

$q_{r}^{(+)} \quad=$ component of radiative flux toward shock, W/m $/ \mathrm{m}^{2}$

$q_{r}^{(-)}$

$q_{\text {Total }}$

$R_{C 1}$

$R_{N}$

$s$

$T$

$\bar{T}$

$T_{\text {REWT }}$

$T_{\text {ref }}$

\section{Nomenclature}

$=$ mass fraction of species $i$

$=$ mass fraction of element $k$

$=$ frozen specific heat of mixture, $\mathrm{J} / \mathrm{kg}-\mathrm{K}$

$=$ binary diffusion coefficient, $\mathrm{m}^{2} / \mathrm{s}$

$=$ enthalpy of undecomposed ablation material, J/kg

$=$ enthalpy of species $i, \mathrm{~J} / \mathrm{kg}$

$=$ enthalpy of species $i, h_{i} / V_{\infty}^{2}$

$=$ thermal conductivity of mixture, $\mathrm{W} / \mathrm{m} \cdot \mathrm{K}$

$=$ thermal conductivity of mixture, $K / \mu_{\text {ref }} C_{P, \infty}$

$=$ Lewis number, $\rho D_{i j} C_{P} / K$

$=$ mass injection rate, $\mathrm{kg} / \mathrm{m}^{2}-\mathrm{s}$

$=$ coordinate measured normal to body, $\mathrm{m}$

$=$ coordinate measured normal to body, $n / R_{C 1}$

$=$ shock standoff distance, $\mathrm{m}$

$=$ Prandtl number, $\mu C_{P} / K$

$=$ pressure, $\mathrm{N} / \mathrm{m}^{2}$

$=$ heat flux, $\mathrm{W} / \mathrm{m}^{2}$

$=$ heat flux, $q / \rho_{\infty} V_{\infty}^{3}$

$=q_{\mathrm{cond}}+q_{\mathrm{conv}}+q_{\mathrm{diff}}, \mathrm{W} / \mathrm{m}^{2}$

$=$ net radiative heat flux in $n$ direction, $q_{r}^{(+)}-q_{r}^{(-)}$, $\mathrm{W} / \mathrm{m}^{2}$

$=q_{c}+q_{r}, \mathrm{~W} / \mathrm{m}^{2}$

$=$ shoulder radius, $\mathrm{m}$

$=$ nose radius, $\mathrm{m}$

= coordinate measured along surface, $\mathrm{m}$

$=$ temperature, $\mathrm{K}$

= temperature, $T / T_{\text {ref }}$

$=$ radiative equilibrium wall temperature, $K$

$=$ reference temperature, $V_{\infty}^{2} / C_{P, \infty}, \mathrm{K}$
Received 21 December 1998; presented as Paper 99-0227 at the AIAA 37th Aerospace Sciences Meeting, Reno, NV, 11-14 January 1999; revision received 2 August 1999; accepted for publication 15 February 2000 . Copyright (C) 2000 by the American Institute of Aeronautics and Astronautics, Inc. No copyright is asserted in the United States under Title 17, U.S. Code. The U.S. Government has a royalty-free license to exercise all rights under the copyright claimed herein for Governmental purposes. All other rights are reserved by the copyright owner.

* Senior Research Engineer, Aero- and Gas-Dynamics Division. Associate Fellow AIAA.

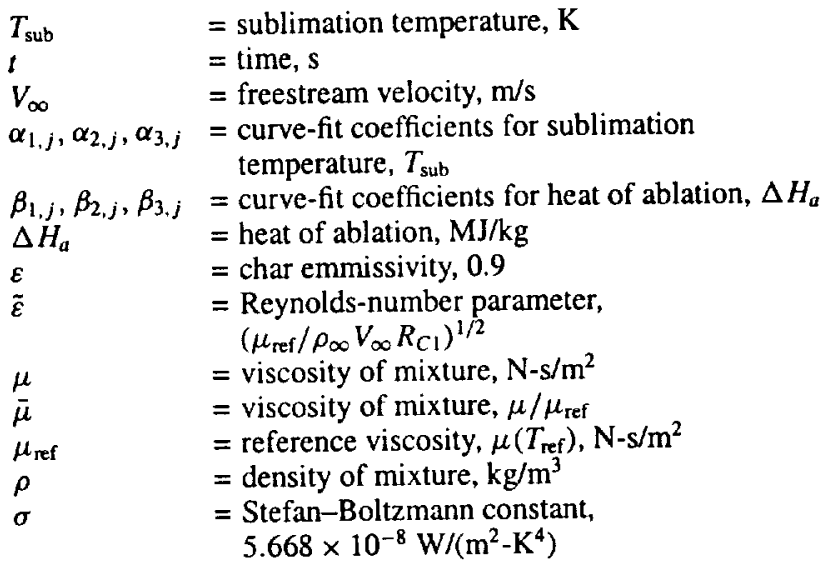

Subscripts

\begin{tabular}{|c|c|}
\hline$A$ & $=$ ablator \\
\hline $\mathrm{abl}$ & $=$ ablation \\
\hline cond & $=$ conduction \\
\hline conv & $=$ convection \\
\hline diff & $=$ diffusion \\
\hline $\mathrm{Eq}$ & $=$ equilibrium \\
\hline$i$ & $=i$ th species \\
\hline$j$ & $=j$ th species \\
\hline$k$ & $=k$ th element \\
\hline$r$ & $=$ radiation \\
\hline$w$ & $=$ wall value \\
\hline- & $=$ values for solid ablation material at surface \\
\hline$\infty$ & $=$ freestream value \\
\hline
\end{tabular}

\section{Introduction}

7 HE Stardust mission, ${ }^{1}$ part of NASA's Discovery Program, plans to fly a spacecraft through the tail of the comet Wild-2 and bring samples of cometary material as well as interstellar dust to Earth for analysis. The collected cometary particles and the dust will be contained in the Stardust Sample Retum Capsule (SRC), which must survive an intense Earth entry heating. At $12.6 \mathrm{~km} / \mathrm{s}$ the SRC entry is the fastest ever attempted into the Earth's atmosphere. This paper focuses on the aerothermodynamic issues concerning the flow environment around the SRC forebody during such an entry.

Since Stardust was scheduled for launch in early 1999, the work presented here was not available in time to impact the SRC design. 
However, a better understanding of the SRC entry environment and the computational tools employed for its analysis will help in promoting a better and more efficient design of the thermal protection system (TPS) for future sample return vehicles such as MUSES-C, Genesis, Champollion (DS-4), and Mars Sample Return. Improved computational tools will also be useful in postflight evaluation of the TPS and other measured quantities.

\section{Previous Work}

Recently, calculations have been done ${ }^{2}$ for the Stardust SRC forebody TPS with an axisymmetric Navier-Stokes flow solver, loosely coupled to the radiation and ablation modules. The thermochemical nonequilibrium flowfield calculations with ablation are based on an 18-species chemical model. The ablation boundary condition (namely, the blowing rate, species mass fractions, and wall temperature) for the flowfield solution are obtained iteratively by assuming the surface composition to be in equilibrium at the temperatures and pressures predicted from a material response code (with inputs of wall heat transfer rate and pressure from the flowfield solution).

Employing a methodology similar to that of Ref. 2, Ref. 3 has recently obtained stagnation-point heat transfer rates for the PioneerVenus probes, ${ }^{4}$ whose flight environment resembles that of current sample retum vehicles. ${ }^{2,5,6}$ A 17-species nonequilibrium chemistry model is used for the shock-layer flow. The pyrolysis gas composition at the surface is obtained by assuming that the surface is in equilibrium at the local temperature and pressure.

A recent analysis ${ }^{7}$ of the MUSES-C asteroid sample return mission has considered a 19-species nonequilibrium chemistry model (with the thermal equilibrium assumption) both for the shock-layer flow and the ablator surface for a true ablation calculation. The chemistry model consists of 11 air species and 8 carbon-containing species. The hydrocarbon species are not included, and the pyrolysis process is not considered to keep the analysis simple. Because the 19-species finite rate chemistry model is implemented both at the surface and in the shock layer, it includes all of the species considered throughout the computational domain. Thus, the analysis is consistent both at the surface and through the shock layer for the chemistry model considered.

\section{Present Work}

In the present work the Stardust SRC entry flowfield is investigated by assuming complete thermal equilibrium. Fully coupled radiation solutions with and without ablation injection are obtained by using an axisymmetric viscous shock-layer method by assuming chemical equilibrium both in the flowfield and at the surface. The elemental continuity equations are solved iteratively for each element to determine the appropriate mix of ablative and freestream elemental composition at the surface (adjacent to the ablator) and through the flowfield. With information concerning the elemental composition, pressure, and temperature, the species concentration at the surface (adjacent to the ablator) and through the flowfield is obtained by using the free energy minimization procedure.

With the calculation of both the flowfield and surface ablation species from the equilibrium assumption, the transition from ablation to freestream elements (and the corresponding species) is obtained continuously ${ }^{8}$ through the solution of elemental continuity equations. Further, the fully equilibrium calculations also bypass the entire discussion about governing processes and intermediate steps concerning the number of species, reaction mechanisms, and the associated reaction rates, especially for the complex flowfields with ablation. Therefore, the flowfield calculations with the thermochemical equilibrium model would appear adequate for the TPS design (for the convective-dominated heating environment), especially if the ablation boundary condition is specified with the equilibrium assumption. Although a nonequilibrium overall solution including surface and flowfield would be desirable, a full equilibrium calculation further provides a conservative estimate of the surface heating with the desired safety factor for the TPS design.

Computed results for an ablating surface include those with the laminar flow assumption as well as those for a fully turbulent flow immediately downstream of the stagnation line. For a nonablating surface results have been obtained with nonequilibrium chemistry and fully catalytic ${ }^{2}$ as well as equilibrium catalytic wall boundary condition. Only at lower temperatures (i.e., $T<2000 \mathrm{~K}$ ) would a fully catalytic boundary condition (with complete recombination to freestream value) be realistic to use.

\section{Analysis}

\section{Flowfield Model}

The viscous shock-layer (VSL) equations employed are those of a multicomponent reacting-gas mixture under conditions of chemical nonequilibrium ${ }^{9}$ and equilibrium ${ }^{8.10}$ with thermal equilibrium. These equations are the same as those given in Refs. 9 and 10, and, therefore, they are not given here. The chemistry model, boundary conditions, and thermodynamic and transport properties employed are similar to those of Refs. 9-11, whereas the ablation injection, radiative transport, and turbulence models (used with equilibrium chemistry only) are those of Refs. 8 and 10-13. These models, boundary conditions, and the properties are briefly described here.

\section{Chemistry Model}

For calculations of airflow over a nonablating surface, an 11-species $\left(\mathrm{N}_{2}, \mathrm{O}_{2}, \mathrm{~N}, \mathrm{O}, \mathrm{NO}, \mathrm{NO}^{+}, \mathrm{e}^{-}, \mathrm{N}^{+}, \mathrm{O}^{+}, \mathrm{N}_{2}^{+}\right.$, and $\left.\mathrm{O}_{2}^{+}\right)$chemistry model is used for nonequilibrium calculations, whereas a 9-species $\left(\mathrm{N}_{2}, \mathrm{O}_{2}, \mathrm{~N}, \mathrm{O}, \mathrm{NO}, \mathrm{NO}^{+}, \mathrm{e}^{-}, \mathrm{N}^{+}\right.$, and $\left.\mathrm{O}^{+}\right)$chemical model is used for the equilibrium flow. For the equilibrium ablation injection calculation, 20 chemical species are used: the 7 equilibrium air species (without $\mathrm{NO}$ and $\mathrm{NO}^{+}$) plus $\mathrm{C}, \mathrm{C}_{2}, \mathrm{C}_{3}, \mathrm{CO}, \mathrm{CN}, \mathrm{C}_{2} \mathrm{H}, \mathrm{C}_{3} \mathrm{H}, \mathrm{C}_{4} \mathrm{H}$, $\mathrm{C}_{2} \mathrm{H}_{2}, \mathrm{C}^{+}, \mathrm{H}, \mathrm{H}_{2}$, and $\mathrm{HCN}$. The equilibrium composition is determined (for a given temperature, pressure, and elemental composition) by using the free-energy minimization method of Ref. 14.

\section{Boundary Conditions}

The boundary conditions at the shock are obtained by using the Rankine-Hugoniot relations. The flow behind the shock is assumed to be in chemical equilibrium or frozen at the freestream composition for equilibrium and nonequilibrium calculations, respectively. ${ }^{9}$ No-slip continuum boundary conditions are employed at the surface. The surface temperature with no ablation injection is assumed to be the radiative equilibrium wall value obtained from

$$
T_{\mathrm{REWT}}=\left\{\left[\bar{q}_{r}^{(-)}+\tilde{\varepsilon}^{2}\left(\bar{K} \frac{\partial \bar{T}}{\partial \bar{n}}+\bar{\mu} \frac{L e}{P_{r}} \sum_{i=1}^{\mathrm{NS}} \bar{h}_{i} \frac{\partial C_{i}}{\partial \bar{n}}\right)\right] \frac{\rho_{\infty} V_{\infty}^{3}}{\varepsilon \sigma}\right\}^{\frac{1}{4}}
$$

For ablation injection cases steady-state ablation is assumed. However, the species surface concentrations, ablation rates, and surface temperatures, in general, can be obtained from a material response code (such as FIAT of Ref. 2), by employing input surface heat flux and pressure from an equilibrium flowfield code. For the surface ablation cases considered in the present study, an energy balance at the flowfield-ablator interface gives the coupled mass injection rate for quasi-steady ablation:

$$
\dot{m}=\left[\frac{-q_{r, w}-q_{r, w}}{\sum_{i=1}^{N S}\left(C_{i} h_{i}\right)_{w}-h_{a}}\right]
$$

The surface temperature for the present calculations with ablation injection is that at which the quasi-steady ablation occurs. The expression used for surface temperature for the Phenolic Impregnated Ceramic Ablator (PICA) ${ }^{15}$ has been obtained by curve fitting these values (computed from the charring material and ablation thermal ${ }^{16.17}$ response code) in the pressure range $0.001 \mathrm{~atm}$ $<p<1.00 \mathrm{~atm}$. The elemental composition of PICA is similar to that of a carbon-phenolic ablator. It is, however, less dense and has much lower thermal conductivity. The expressions for the sublimation temperature and heat of ablation for PICA (with $92 \%$ carbon, $4.9 \%$ oxygen, $2.2 \%$ hydrogen, and $0.9 \%$ nitrogen by mass) are

$$
\begin{aligned}
T_{\mathrm{abl}} & =\sum_{j=1}^{5} \alpha_{1, j}\left(\log C_{A}\right)^{j-1}+\left(\log p_{w}\right) \sum_{j=1}^{5} \alpha_{2, j}\left(\log C_{A}\right)^{j-1} \\
& +\left(\log p_{w}\right)^{2} \sum_{j=1}^{5} \alpha_{3, j}\left(\log C_{A}\right)^{j-1}
\end{aligned}
$$


Table 1 Coefficients for ablation temperature for PICA

\begin{tabular}{lccccc}
\hline \hline & \multicolumn{5}{c}{$j$} \\
\cline { 2 - 6 } Coefficients & 1 & 2 & 3 & 4 & 5 \\
\hline$\alpha_{1, j}$ & 3790.0 & 86.795 & -2980.0 & -8250.2 & -7631.7 \\
$\alpha_{2, j}$ & 329.94 & -66.703 & -1524.6 & -4340.9 & -3885.7 \\
$\alpha_{3, j}$ & 20.386 & -17.654 & -268.62 & -771.00 & -684.89 \\
\hline \hline
\end{tabular}

where $p_{w}$ is the wall pressure in atmospheres and $C_{A}$ is the ablator mass fraction at the surface. The values for $\alpha_{1, j}$ are given in Table 1 . For the case when the gas species adjacent to the surface are caused solely by the ablation species (i.e., $C_{A}=1.0$ ), Eq. (3) with the values of $\alpha_{1, j}$ in Table 1 gives

$$
T_{\mathrm{abl}}=3790.0+329.94\left(\log p_{w}\right)+20.386\left(\log p_{w}\right)^{2}
$$

The surface temperature and the coupled mass injection rate are calculated by iterating the solution of the governing flowfield equations and the boundary conditions.

For ablation injection the elemental concentrations at the wall are governed by convection and diffusion and are obtained from the equation

$$
\left(\frac{\partial \tilde{C}_{k}}{\partial \bar{n}}\right)_{w}-\frac{1}{\tilde{\varepsilon}^{2}}\left(\frac{\dot{m} P r}{\mu L e}\right)_{w}\left[\left(\tilde{C}_{k}\right)_{w}-\left(\tilde{C}_{k}\right)_{-}\right]=0
$$

For the radiative transport calculations the bow shock is considered transparent, and the freestream is considered cold and transparent. Therefore, the precursor effects are neglected. Further, the body surface is assumed to be gray with a reflectivity of 0.1 , emissivity of 0.9 , and transmissivity of 0 . The energy reradiated from the surface is included both in the radiation transport calculation as well in the surface energy balance [Eq. (2)]. The net radiative flux can be represented as

$$
q_{r}=q_{r}^{(+)}-q_{r}^{(-)}
$$

At the surface

$$
q_{r, w}^{(+)}=\varepsilon \sigma T_{w}^{4}
$$

The heat transferred to the wall because of conduction, diffusion, and convection is

$$
\begin{aligned}
-q_{c, w} & =\tilde{\varepsilon}^{2} \rho_{\infty} V_{\infty}^{3}\left[\bar{K} \frac{\partial \bar{T}}{\partial \bar{n}}+\bar{\mu} \frac{L e}{\operatorname{Pr}} \sum_{i=1}^{\mathrm{NS}} \bar{h}_{i} \frac{\partial C_{i}}{\partial \bar{n}}\right. \\
& \left.-\frac{\dot{m}}{\rho_{\infty} V_{\infty}} \sum_{i=1}^{\mathrm{NS}}\left(C_{i} \bar{h}_{i}-C_{i-} \bar{h}_{i-}\right)_{w}\right]
\end{aligned}
$$

where NS is the number of species.

\section{Catalytic Wall Conditions}

For nonablating, nonequilibrium flow the following three catalytic wall boundary conditions are used.

1) Noncatalytic wall (NCW): Because no reactions occur at the surface in this case, the mass-fraction gradients for all species are zero at the surface, i.e.,

$$
\left(\frac{\partial C_{i}}{\partial \vec{n}}\right)_{w}=0
$$

2) Equilibrium catalytic wall (ECW): The wall catalyzed reactions are assumed to occur at an infinite rate, and, therefore, the species mass fractions at the wall are those corresponding to their local equilibrium values, i.e.,

$$
\left(C_{i}\right)_{w}=\left(C_{i}\right)_{\mathrm{Eq}}=f\left(p_{w}, T_{w}\right)
$$

3) Fully catalytic wall (FCW): The gas species at the surface are assumed to recombine to the freestream composition, i.e.,

$$
\left(C_{i}\right)_{w}=\left(C_{i}\right)_{\infty}
$$

At low surface temperatures the surface condition of Eq. (10) reduces to Eq. (11).

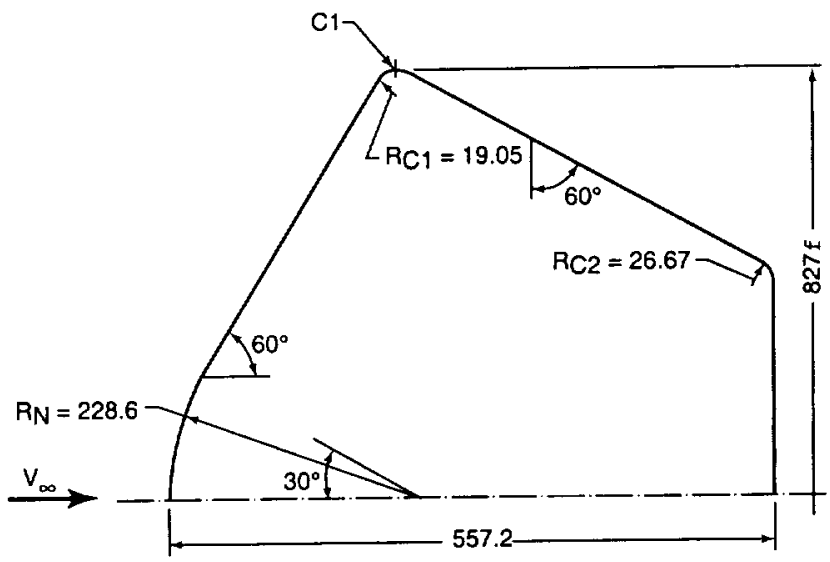

Fig. 1 Geometry of SRC; dimensions in millimeters.

Radiative Transport

The radiation transport code RADICAL ${ }^{18,19}$ has been used to compute radiative heat flux $q_{r}$. This code accounts for the effects of nongray self-absorption and includes the molecular band, continuum, and atomic line transitions. The ultraviolet properties for $C_{3}$ are taken from Ref. 20 . The radiative transport is fully coupled with the flowfield solutions for equilibrium chemistry. The nonequilibrium total heat transfer rate (for a nonablating surface) is obtained by adding the equilibrium radiative component to the nonequilibrium conductive and diffusive components.

\section{Thermodynamics and Transport Properties}

Thermodynamic properties for specific heat, enthalpy and free energy, and transport properties for viscosity and thermal conductivity are required for each species considered. Values of these properties are obtained by using polynomial curve fits of Refs. 8 and 11. The equilibrium composition is determined by a free-energy minimization calculation procedure of Ref. 14. Mixture viscosity is obtained by the method of Armaly and Sutton, ${ }^{21}$ and mixture thermal conductivity is computed by the Mason and Saxena ${ }^{22}$ relation. Both constant and variable Lewis number values are employed in the computations. However, the results presented here are for a variable Lewis number based on the effective diffusion coefficient $D_{m}$ for the mixture. ${ }^{8}$

\section{Turbulence Model}

A two-layer, eddy-viscosity, Cebeci-Smith turbulence mod$\mathrm{el}^{13,23,24}$ is employed in the present investigation. Reference 24 gives a detailed description of the model and various expressions for it. The boundary-layer edge definition used in the current study is based on an index of diffusion, conduction, and dissipation. ${ }^{25}$ The transition to turbulent flow is assumed to occur instantaneously at the first grid point downstream of the stagnation point. The turbulent Prandtl and Lewis numbers are assumed to be 0.9 and 1.0 , respectively.

Solution Procedure

The method used to solve the nonequilibrium and equilibrium VSL equations is a spatial-marching, implicit, finite-difference technique, ${ }^{8,13}$ which includes coupling of the global continuity and normal momentum equations and use of the Vigneron pressure condition in the subsonic region (which covers a large part of the forebody of Stardust capsule shown in Fig. 1). The shoulder radius $R_{C l}$ is employed for the reference length in place of the conventionally used nose radius $R_{N}$. Details of the method of solution are similar to those of Refs. 8 and 13 and, therefore, are not presented here.

\section{Results and Discussion}

Results are presented for the forebody of the SRC shown in Fig. 1. The overshoot entry trajectory ${ }^{2}$ (which produces maximum heat loads) used in the calculations is given in Fig. 2. The freestream conditions at the calculation points for this trajectory are provided in Table 2. Peak heating and pressure occur at approximately 54 and $66 \mathrm{~s}$, respectively, for this trajectory. The SRC forebody is an 
Table 2 Freestream conditions for Stardust entry trajectory

\begin{tabular}{lcccc}
\hline \hline $\begin{array}{l}\text { Time, } \\
\mathrm{s}\end{array}$ & $\begin{array}{c}\text { Altitude, } \\
\mathrm{km}\end{array}$ & $\begin{array}{c}\text { Velocity, } \\
\mathrm{m} / \mathrm{s}\end{array}$ & $\begin{array}{c}\text { Density, } \\
\mathrm{kg} / \mathrm{m}^{3}\end{array}$ & $\begin{array}{c}\text { Temperature, } \\
\mathrm{K}\end{array}$ \\
\hline 34.00 & 81.64 & $12,590.4$ & $9.63 \times 10^{-6}$ & 216.93 \\
42.00 & 71.92 & $12,413.4$ & $1.29 \times 10^{-5}$ & 221.42 \\
48.00 & 65.44 & $12,004.0$ & $1.06 \times 10^{-4}$ & 229.00 \\
54.00 & 59.77 & $11,136.7$ & $2.34 \times 10^{-4}$ & 238.47 \\
60.00 & 55.02 & $9,718.7$ & $4.39 \times 10^{-4}$ & 248.48 \\
66.00 & 51.19 & $7,956.9$ & $7.21 \times 10^{-4}$ & 253.55 \\
76.00 & 46.51 & $5,178.9$ & $1.35 \times 10^{-3}$ & 256.90 \\
\hline \hline
\end{tabular}

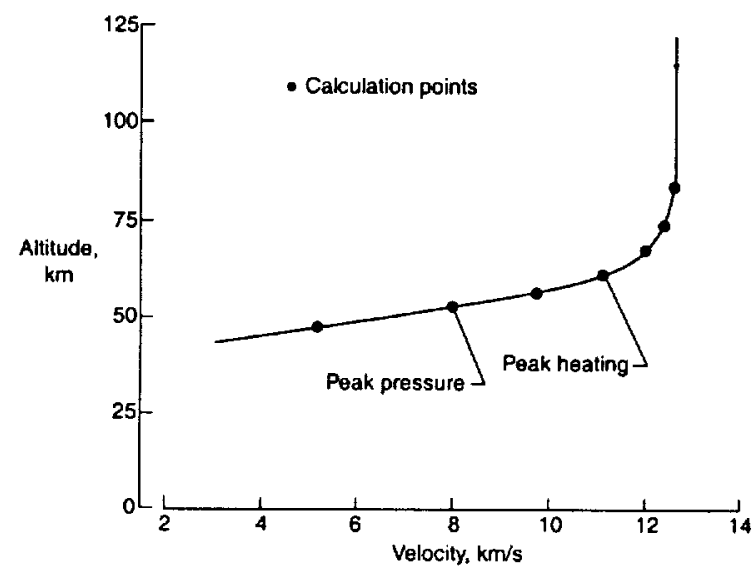

Fig. 2 Stardust entry trajectory.

axisymmetric $60-\mathrm{deg}$ sphere cone with a nose radius $R_{N}$ of approximately $23 \mathrm{~cm}$. The computational domain for the present VSL results extends to just past the highest point $C 1$ on the shoulder, where the flow is predominantly supersonic. The PICA heatshield for the forebody extends beyond this point. ${ }^{2}$ A $100 \times 125$ grid is employed with all of the computations. Variable grid sizes are used both normal to and along the body surface. The minimum distance between normal grid points is $\left(2 \times 10^{-4}\right) R_{C 1}$. In the direction along the surface, the minimum grid size is $\left(1 \times 10^{-1}\right) R_{C 1}$ on the shoulder to resolve the flowfield there and is as large as five times this value in the nose region to reduce the computational time. These values of the grid sizes have been established to ensure grid independence of the solution at peak heating condition $(t=54 \mathrm{~s})$. Calculations were done by using the Cray C 90 computer. The computational time required is about 200 and $600 \mathrm{CPU} s$ per global pass for the nonequilibrium and equilibrium flow calculations around the body (without radiation), respectively. Typically, two global passes are required for convergence of the shock shape and surface heating.

\section{Nonablating Results Along Trajectory with Nonequilibrium} and Equilibrium Chemistry

Nonablating stagnation-point total heat transfer rate (conduction + diffusion + radiation) is provided at different times along the entry trajectory in Fig. 3. Results are obtained with equilibrium as well as nonequilibrium (finite rate) flowfield chemistry. Three surface recombination boundary conditions, namely, FCW, ECW, $\mathrm{NCW}$ are used with the finite rate chemistry calculations. Present results for the FCW case are in good agreement with those obtained by Olynick et al. ${ }^{2}$ up to time $t=60 \mathrm{~s}$. A maximum value of about $1250 \mathrm{~W} / \mathrm{cm}^{2}$ is obtained at $t=54 \mathrm{~s}$ from the present calculations. The corresponding value obtained from Ref. 2 is about $2 \%$ higher. However, the difference between these two values increases for $t>60 \mathrm{~s}$ and may be caused by grid resolution employed in Ref. 2. Further, the present finite rate results with an ECW boundary condition are close to the equilibrium flow results as expected. A maximum value of about $1100 \mathrm{~W} / \mathrm{cm}^{2}$ is obtained at $t=54 \mathrm{~s}$ in this case. The NCW predictions are included for reference and give the lowest surface heating with a maximum value of only about 650 $\mathrm{W} / \mathrm{cm}^{2}$ at $t=54 \mathrm{~s}$.

The radiation component for total nonequilibrium heating is obtained from the corresponding equilibrium calculations and is about $115 \mathrm{~W} / \mathrm{cm}^{2}$ at peak heating $(t=54 \mathrm{~s})$. Figure 4 shows the presently

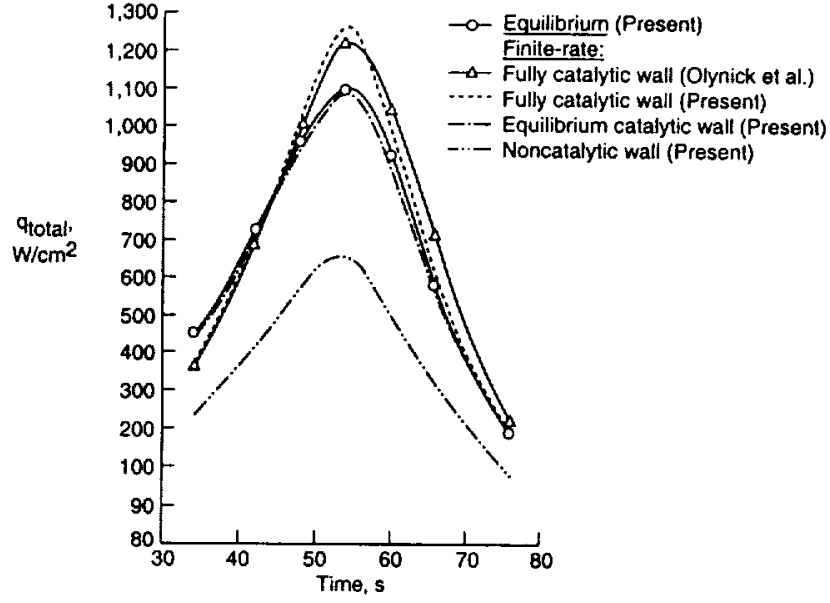

Fig. 3 Nonablating stagnation-point total heat transfer rate.

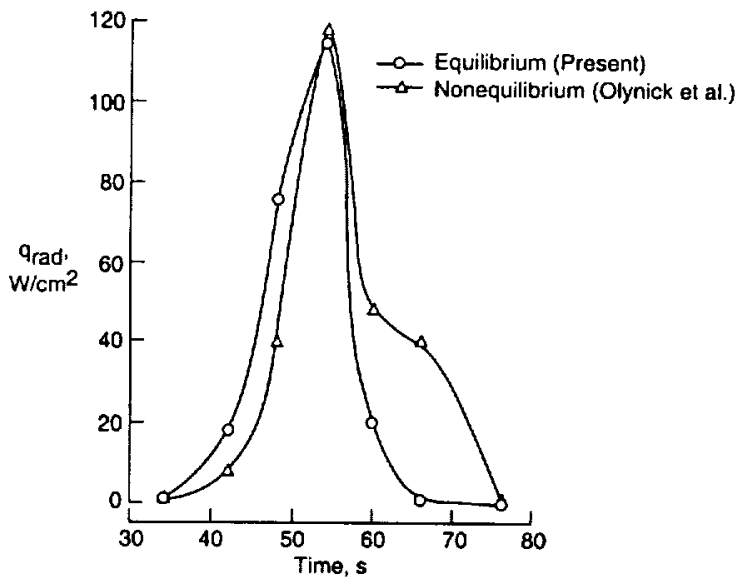

Fig. 4 Nonablating stagnation-point radiative heat transfer rate.

computed stagnation-point equilibrium radiative heat transfer rate as well as the values obtained in Ref. 2 from a nonequilibrium ra. diation calculation. It is not clear why the nonequilibrium radiative heating is lower at earlier times and higher at later times in the trajectory as compared with the equilibrium calculations. Generally, the nonequilibrium effects (which are likely to be present at earlier times in the trajectory) should increase ${ }^{26}$ radiative heating in comparison with the equilibrium value, and it should approach the equilibrium value at later times in the trajectory (with the increasing Reynolds number).

The radiative equilibrium wall temperatures for the heating calculations of Fig. 3 are shown in Fig. 5. A value of 0.9 is used for char emissivity $\varepsilon$ in the present calculations. Similar to the surface heat transfer values, present FCW predictions for surface temperature are in good agreement with those of Olynick et al ${ }^{2}$ Reference 2 employed a value of 1.0 for $\varepsilon$, with zero reflectivity assumed. Figure 5 also shows Olynick's values adjusted for $\varepsilon=0.9$. Differences between the present predictions and those of Ref. 2 increase at a later time in the trajectory for the reasons mentioned earlier. For most of the investigated trajectory, the surface temperatures are greater than $3000 \mathrm{~K}$. Consequently, the FCW boundary condition is physically inappropriate because full recombination of air (for FCW boundary condition) cannot be forced for temperatures greater than about $2000 \mathrm{~K}$. A physically appropriate surface recombination condition for these temperatures is a finite catalytic wall condition, which would be bounded by the ECW (most conservative) and the NCW boundary conditions. A maximum value of about $3800 \mathrm{~K}$ is obtained at $t=54 \mathrm{~s}$ for the present finite rate results with an ECW condition. These results are close to those obtained with the equilibrium flowfield chemistry as expected. The NCW calculations give the lowest surface temperatures as noted with the surface heating results of Fig. 3. 


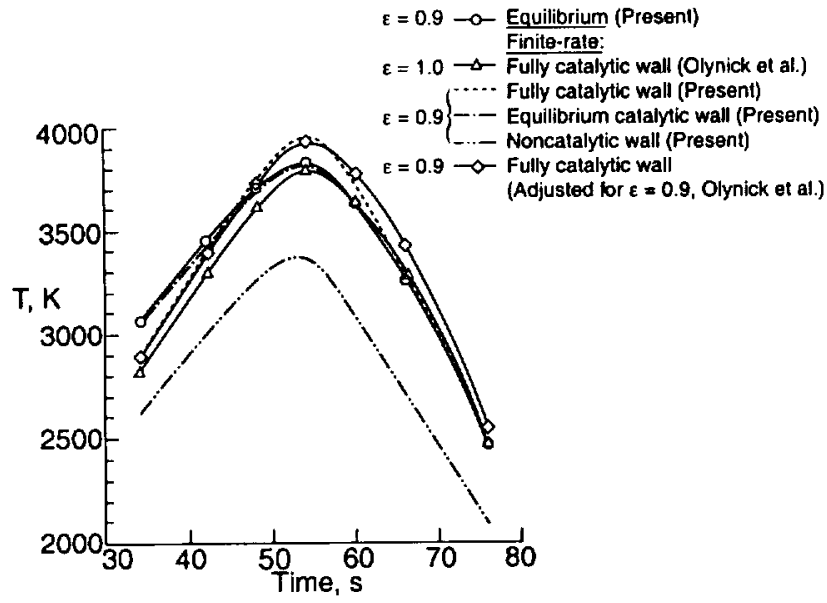

Fig. 5 Nonablating stagnation-point temperature.

Ablation Results Along Trajectory with Equilibrium Chemistry

Figure 6a shows the equilibrium stagnation total heat transfer rate with and without ablation along the trajec.ory. Ablation produces a $35 \%$ reduction in the heating at peak-heating time of $t=54 \mathrm{~s}$. The corresponding peak stagnation heat transfer rate of Ref. 2 (without ablation) is about $10 \%$ higher than the present value, and their results also show a reduction of about $35 \%$ with ablation. Components of the total heat transfer rates of Fig. 6a are shown in Fig. 6b. Reduction in the convective component $q_{c}$ [which is given by Eq. (8) and consists of conduction, diffusion, and convection] by ablation is caused by injection cooling. Ablation injection reduces the surface gradients of temperature and that of various species mass fractions; this causes a decrease in the conductive and diffusive heat fluxes. The radiative component, which is relatively small (less than $11 \%$ of the total heating without ablation), is not impacted much by ablation injection. There is a slight increase in radiation with ablation before the peak heating ( $t=54 \mathrm{~s}$ ). There is a deeper penetration of the shock layer by the ablation species $\mathrm{C}$ and $\mathrm{CO}$ during earlier times in the trajectory, and the increase in radiation from $\mathrm{C}$ line and $\mathrm{CO}\left(4^{+}\right)$molecular contributions is only partially offset by the absorption of radiation by ablation species during that period.

Surface temperatures used with the equilibrium stagnation heating calculations are given in Fig. 7. Surface heating without ablation is obtained by using the radiative equilibrium wall temperature as already mentioned (see Fig. 5), whereas the ablation temperature from Eq. (3) is used for the ablation injection calculations. The no ablation temperatures are generally higher (because of the higher surface heating) than those with ablation. Present ablation temperature values are close to those obtained by Olynick et al. ${ }^{2}$ up to the peak heating time $(t<54 \mathrm{~s})$ in the trajectory. At later times the present surface temperature values are lower by a maximum of about $18 \%$ (at $t=76 \mathrm{~s}$ ). Also shown in Fig. 7 is the mass fraction of ablation species at surface, with a maximum value of about 0.25 at peak heating time $(t=54 \mathrm{~s})$. This value implies that $75 \%$ of the mass at the surface is from the freestream at that time in the trajectory.

The surface ablation injection rate along the trajectory as well as the ratio of injection rate to the freestream mass flux, corresponding to the heat transfer rate of Fig. 6a, are shown in Fig. 8. A maximum value of $3 \%$ of the ratio is obtained at time $t=34 \mathrm{~s}$. The value of this ratio decreases to about $1.5 \%$ at peak heating, where the maximum injection rate of about $0.04 \mathrm{~kg} / \mathrm{m}^{2}-\mathrm{s}$ is obtained. Even though similar reduction ( $35 \%$ ) in heating is obtained with ablation, presently computed values of the injection rate and injection mass flux ratio at peak heating ( $t=54 \mathrm{~s})$ are about one-half of those obtained in Ref. 2 . These differences are believed to be caused by the differences in the mass fraction of ablation species and their enthalpies used in the two calculations. As pointed out in the next section, some of the species identified as significant in the present work are not included in the equilibrium surface analysis and fowfield chemical kinetics model of Ref. 2

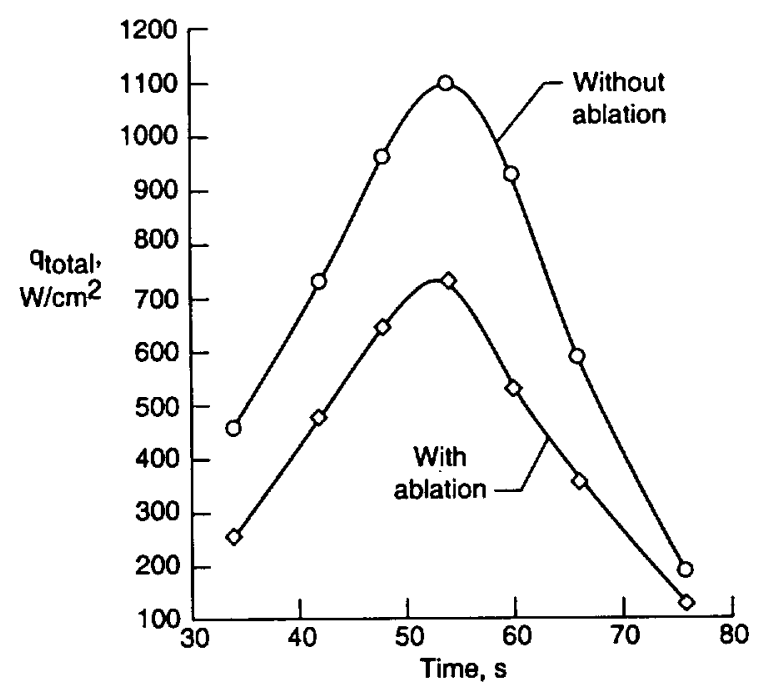

Fig. 6a Equilibrium stagnation-point total heat transfer rate.

$$
\begin{aligned}
& \rightarrow \text { With ablation } \\
& -\infty \text { Without ablatioin }
\end{aligned}
$$

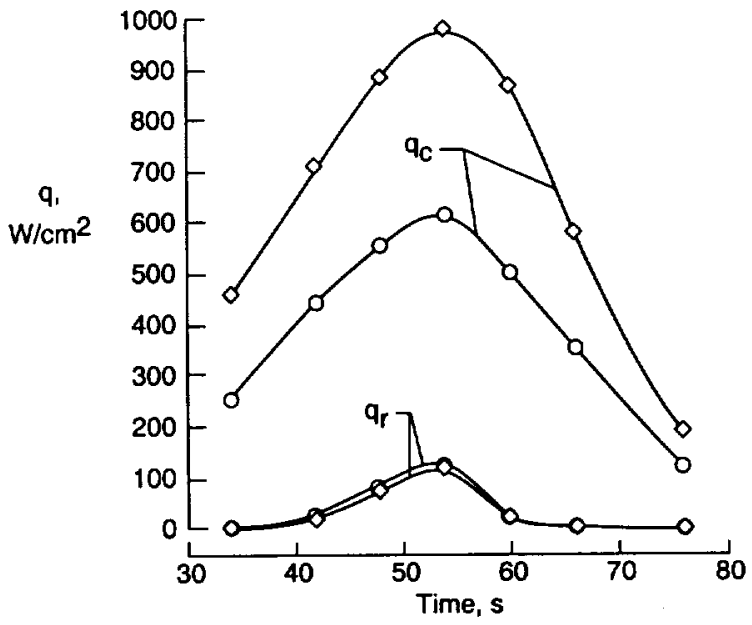

Fig. 6b Components of equilibrium stagnation-point heat transfer rate.

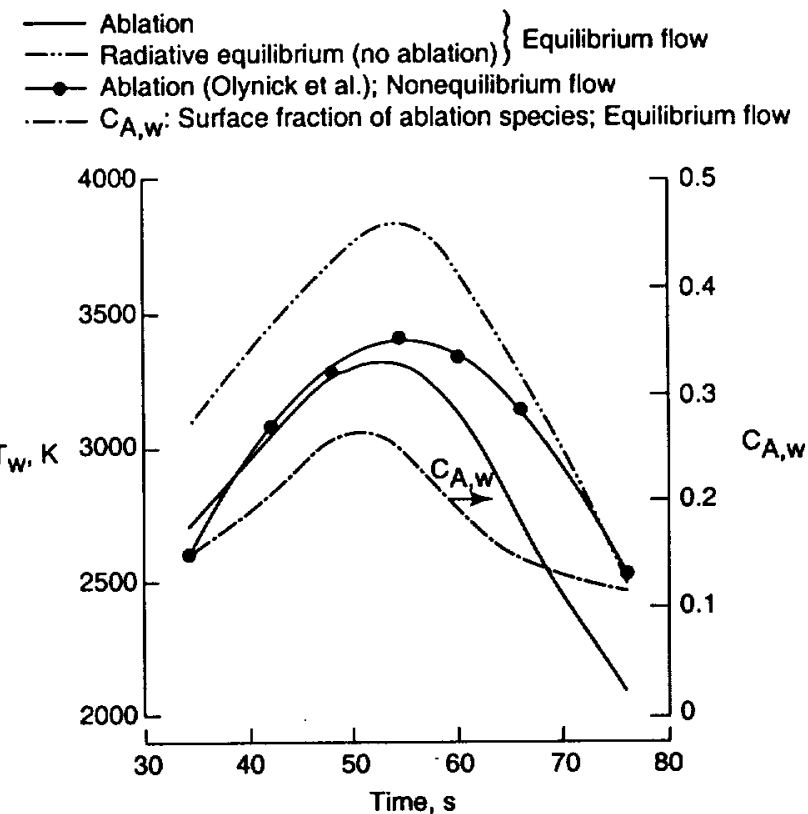

Fig. 7 Stagnation-point surface temperature and ablator mass fraction. 


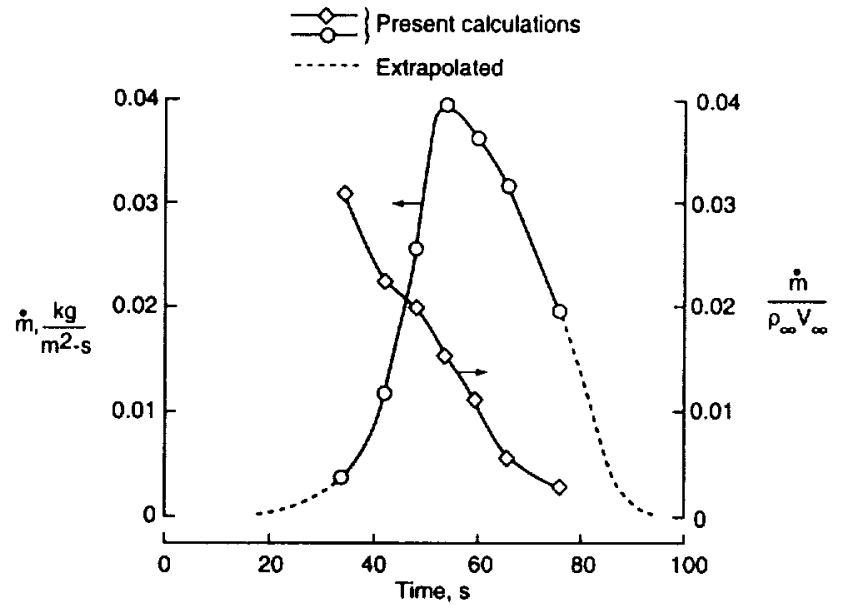

Fig. 8 Equilibrium stagnation-point mass injection rate.

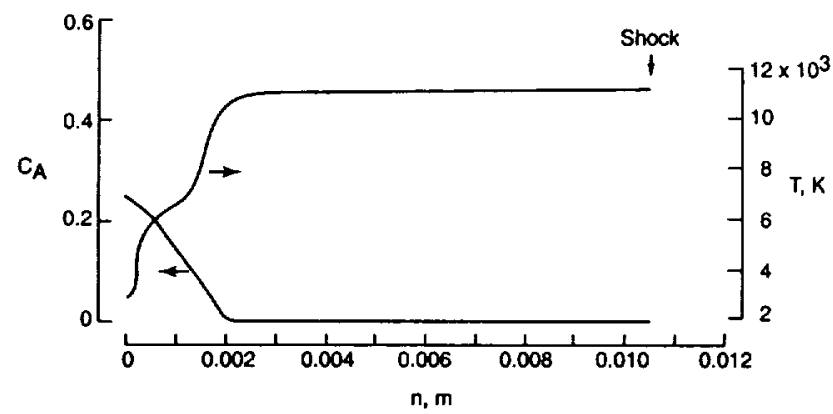

Fig. 9 Stagnation ablator mass fraction and temperature profiles [equilibrium flow; peak heating $(t=54 \mathrm{~s})$ ].

Laminar/Turbulent Ablation Results at Peak Heating

Condition with Equilibrium Chemistry

Stagnation Profiles

Figure 9 shows the temperature and ablator mass-fraction profiles at peak heating $(t=54 \mathrm{~s})$. The effect of ablation injection is limited to about $20 \%$ of the flowfield close to the surface at that time. This effect is also evident from the mass-fraction profiles of the freestream and ablation species shown in Fig. 10. The dominant ablation species not included in Ref. 2 are $\mathrm{C}_{2} \mathrm{H}, \mathrm{C}_{3} \mathrm{H}$, and $\mathrm{C}_{4} \mathrm{H}$. Noninclusion of these species can be partly responsible for the differences in present mass loss rate and those of Ref. 2 as mentioned earlier (see Fig. 8).

\section{Forebody Surface Distributions}

Surface distributions of the total heat flux for laminar and fully turbulent flow cases over the forebody of the Stardust Capsule are given in Fig. 11. This figure shows the effect of both ablator (PICA) mass injection and turbulence on total heating distributions for the peak-heating time of $t=54 \mathrm{~s}$ along the trajectory. The coupled PICA mass injection distributions are shown in Fig. 12. The impact of ablation injection on total heating is very pronounced for the laminar flow. In this case the total heating is reduced by about $35 \%$ along the forebody (compared with the nonablating surface), essentially through the reduction of the convective component as discussed earlier for the stagnation point. However, for the turbulent solutions, where the flow is assumed to undergo instantaneous transition at $s / R_{N}$ equal to 0.05 , the reduction in total heating is less than $13 \%$ as compared with the nonablating laminar flow value on both the conical flank and shoulder. Obviously the benefit of ablation injection in reducing the heating for the laminar flow is partially negated when the flow is assumed to be turbulent. The mass injection rate distributions of Fig. 12, in general, follow the surface heat-flux distributions of Fig. 11. The stagnation nondimensional injection rate $\dot{m} / \rho_{\infty} V_{\infty}$ of 0.015 corresponds to a dimensional value of about $0.040 \mathrm{~kg} / \mathrm{m}^{2}-\mathrm{s}$ (as noted earlier).

There is no noticeable effect of ablation injection and turbulence on surface pressure distribution as shown in Fig. 13. The stagnation

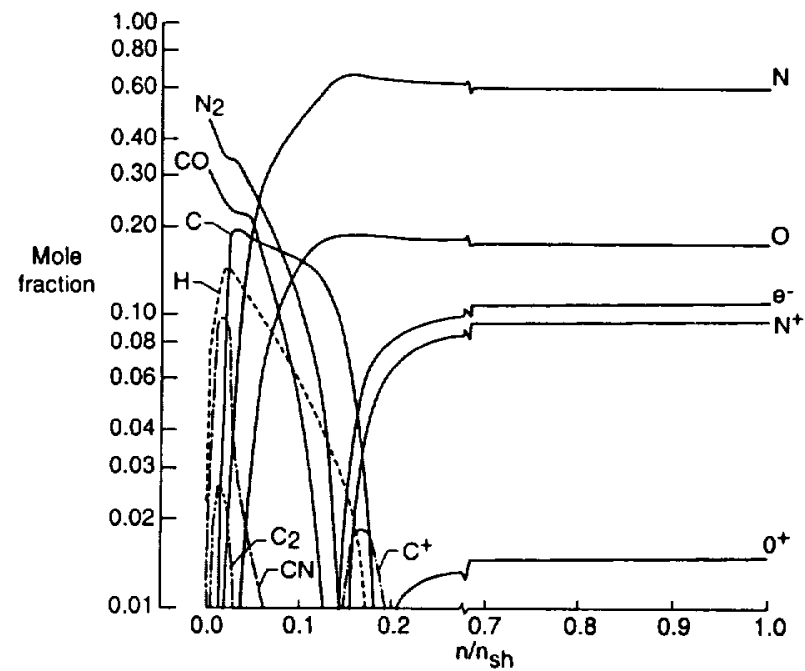

Fig. 10a Stagnation freestream and ablation species profiles [equilibrium flow; peak heating $(t=54 \mathrm{~s}), n_{\mathrm{sh}}=0.0105 \mathrm{~m}$ ).

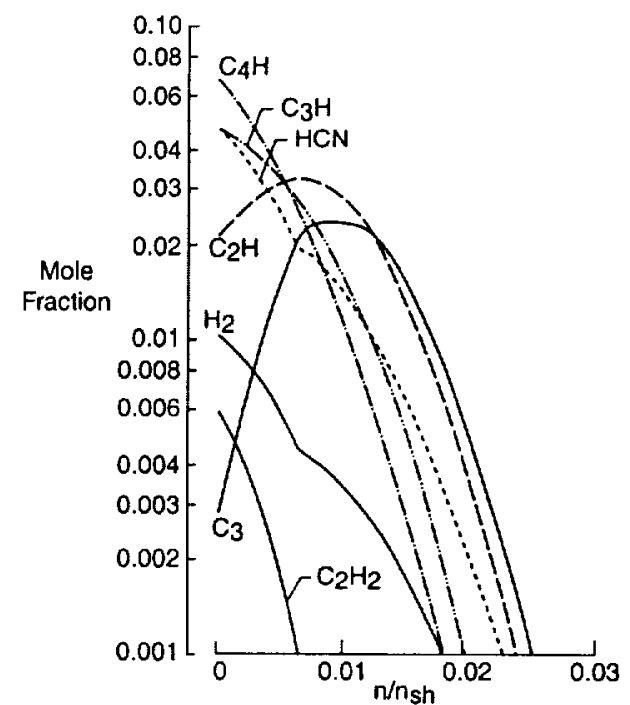

Fig. 10b Stagnation ablation species profiles [equilibrium flow; peak heating $(t=54 \mathrm{~s}), n_{\mathrm{sh}}=0.0105 \mathrm{~m}$ ).

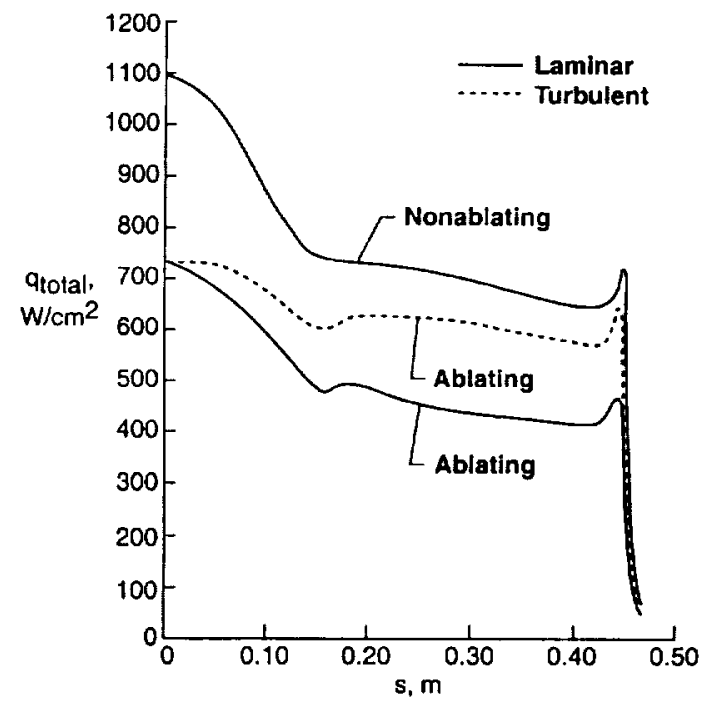

Fig. 11 Total surface heat-flux distribution [equilibrium flow; peak heating $(t=54 \mathrm{~s})]$. 


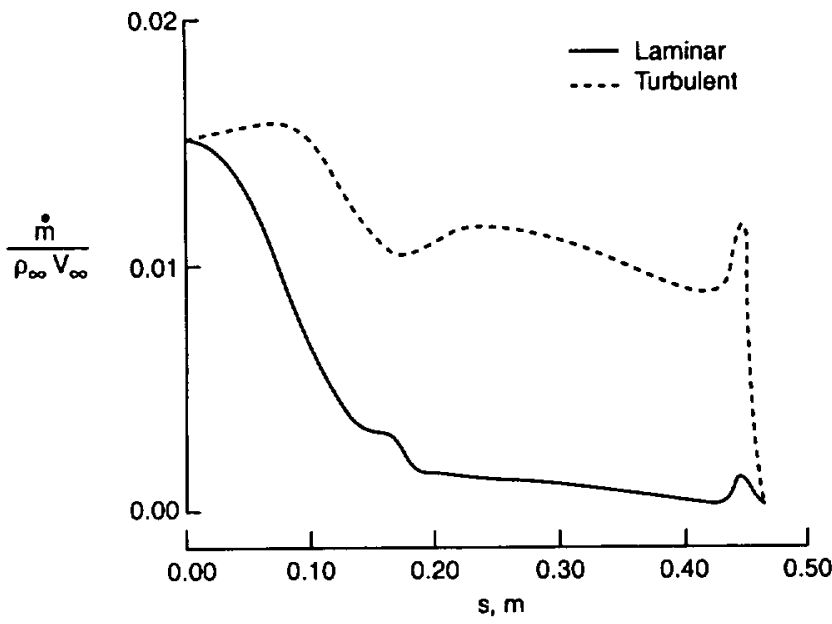

Fig. 12 Mass injection rate distribution [equilibrium flow; peak heating $(t=54 \mathrm{~s})]$.

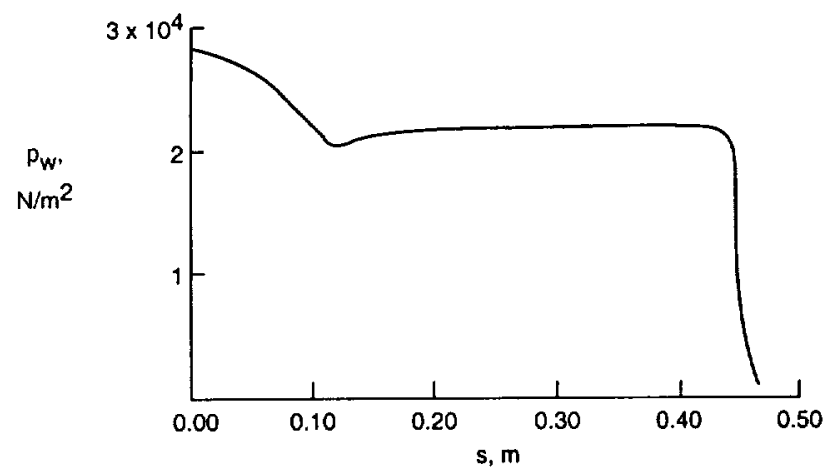

Fig. 13 Surface pressure distribution (nonablating/ablating) [equilibrium flow (laminar/turbulent); peak heating $(t=54 \mathrm{~s})]$.

$(s=0)$ pressure in this figure is about $28,000 \mathrm{~N} / \mathrm{m}^{2}$. This value and the pressure distribution are similar to those of Ref. 2 .

\section{Conclusion}

Results are presented for the forebody of the SRC entering the Earth's atmosphere. Solutions are obtained from an axisymmetric VSL analysis with and without surface ablation including the effect of turbulence.

The forebody aeroshell consists of a 60-deg sphere cone with a shoulder radius one-twelfth that of the nose. For proper resolution of the flowfield over the shoulder, the VSL equations are scaled with the shoulder radius in place of the conventionally employed nose radius. These equations are globally iterated with the Vigneron pressure condition to treat the large embedded subsonic region between the stagnation line and the supersonic outflow at the top of the shoulder.

The no-ablation VSL calculations employ an 11 -species nonequilibrium chemistry model. For these calculations an ECW boundary condition is physically consistent and appropriate to use in place of the FCW condition (with complete recombination to the freestream species). The fully coupled ablation injection calculations are done with a 20 -species equilibrium chemistry model. With fully equilibrium calculations the elemental conservation equations are solved iteratively for each element to determine the elemental composition at the surface (adjacent to the ablator) and in the flowfield. In this formulation the species boundary condition problem at the surface encountered with finite rate calculations is avoided for the case when the ablation injection rate is small, and the gas composition at the surface is caused by both the freestream and ablation products. The small injection rates are usually encountered before large-scale oxidation and sublimation drive the species caused by the freestream away from the surface.

The maximum stagnation heating of about $1250 \mathrm{~W} / \mathrm{cm}^{2}$ is obtained without ablation injection with nonequilibrium calculations and complete surface recombination (i.e., FCW boundary condition), whereas a value of about $1100 \mathrm{~W} / \mathrm{cm}^{2}$ is obtained for a more realistic ECW boundary condition with a radiative equilibrium wall temperature of about $3800 \mathrm{~K}$. Stagnation heating similar to the later value is obtained with a fully equilibrium calculation. The maximum value of radiative heating component is about $11 \%$ at peak heating. With ablation injection a decrease of about $35 \%$ in the total stagnation-point heating (with equilibrium chemistry) is obtained at the peak-heating point in the trajectory. Reduction in heating is slightly less downstream of the stagnation point and along the conical flank, including the shoulder for the laminar case. For the turbulent solutions where the flow is assumed to undergo instantaneous transition just downstream of the stagnation line, the heating is reduced by only about $13 \%$ on the conical flank and shoulder as compared with the nonablating laminar flow. Augmentation of the convective heating by turbulence appears to partially negate the benefit of heating reduction caused by ablation injection in this case.

\section{Acknowledgments}

The author would like to gratefully acknowledge the following contributions: K. Sutton obtained the curve fits for ablation temperature used in this work; J. N. Moss helped with the discussion concerning the specification of species boundary condition with small injection rates; and R. A. Mitcheltree provided helpful suggestions during the preparation of the manuscript.

\section{References}

'Atkins, K. L., Brownlee, D. E., Duxbury, T., Yen, C., and Tsou, P., "STARDUST: Discovery's Interstellar Dust and Cometary Sample Return Mission," Proceedings of the IEEE, Vol. 4, Feb. 1997, pp. 229-245.

${ }^{2}$ Olynick, D., Chen, Y.-K., and Tauber, M., "Aerodynamics of the Stardust Sample Return Capsule," Journal of Spacecrafi and Rockets, Vol. 36, No. 3, 1999, pp. 442-451.

${ }^{3}$ Park, C., and Ahn, H. K., "Stagnation-Point Heat Transfer Rates for Pioneer-Venus Probes," Journal of Thermophysics and Heat Transfer, Vol. 13, No. 1, 1999, pp. 33-41.

${ }^{4}$ Wakefield, R. M., and Pitts, W. C., "Analysis of the Heat-Shield Experiment on the Pioneer-Venus Entry Probes," AIAA Paper 80-1494, July 1980.

${ }^{5}$ Ahn, H. K., and Park, C. "Preliminary Study of the MUSES-C Reentry," AIAA Paper 97-0278, Jan. 1997.

${ }^{6}$ Ahn, H. K., Park, C., and Sawada, K., "Dynamics of Pyrolysis Gas in Charring Materials Ablation," AIAA Paper 98-0165, Jan. 1998.

${ }^{7}$ Suzuki, K., Kubota, H., Fujita, K., and Abe, T., "Chemical Nonequilibrium Stagnation Ablation Analysis of MUSES-C Super-Orbital Re-Entry Capsule," Journal of Spacecraft and Rockets, Vol. 35, No. 3, 1998, pp. 407409.

${ }^{8}$ Gupta, R. N., Lee, K. P., and Scott, C. D., "Aerothermal Study of Mars Pathfinder Aeroshell," Journal of Spacecraft and Rockets, Vol. 33, No. 1, 1996, pp. 61-69.

${ }^{9}$ Gupta, R. N., Lee, K.-P., and Zoby, E. V., "Enhancements to ViscousShock-Layer Technique," Journal of Spacecraft and Rockets, Vol. 30, No. 4, 1993, pp. 404-413.

${ }^{10}$ Moss, J. N., Simmonds, A. L., and Anderson, E. C., "Turbulent Radiating Shock Layers with Coupled Ablation Injection," Journal of Spacecraf and Rockets, Vol. 17, No. 3, 1980, pp. 177-183.

${ }^{11}$ Gupta, R. N., Yos, J. M., Thompson, R. A., and Lee, K. P., "A Review of Reaction Rates and Thermodynamic and Transport Properties for an 11-Species Air Model for Chemical and Thermal Nonequilibrium Calculations to 30,000 K," NASA KP-1232, Aug. 1990.

${ }^{12}$ Gupta, R. N., Moss, J. N., Zoby, E. V., and Simmonds, A. L., "An Evaluation of Turbulence Models for Massively Blown Surfaces," Entry Vehicle Heating and Thermal Protection Systems, edited by P. E. Bauer and H. E. Collicott, Vol. 85, Progress in Astronautics and Aeronautics, AIAA, New York, 1983, pp. 446-471.

${ }^{13}$ Gupta, R. N., Lee, K. P., Zoby, E. V., Moss, J. N., and Thompson, R. A. "Hypersonic Viscous Shock-Layer Solutions over Long Slender BodiesPart I: High Reynolds Number Flows," Journal of Spacecraft and Rockets, Vol. 27, No. 2, 1990, pp. 175-184.

${ }^{14}$ Stroud, C. W., and Brinkley, K. L., "Chemical Equilibrium of Ablation Materials Including Condensed Species," NASA TN D-5391, Aug. 1969.

${ }^{15}$ Tran, H. Johnson, C., Rasky, D., Hui, F., Chen, Y. K., and Hsu, M., "Phenolic Impregnated Carbon Ablators (PICA) for Discovery Class Mission" AIAA Paper 96-1911, June 1996.

${ }^{16}$ Bartlett, E. P., and Kendall, R. M., "An Analysis of the Coupled Chemically Reacting Boundary Layer and Charring Ablator," Pt. 3, NASA CR1062, June 1968. 
17“Aerotherm Charring Material Thermal Response and Ablation Program (CMA 87S)," Acurex Corp., Rept. UM-87-13/ATD, Mountain View, CA, Nov. 1987.

${ }^{18}$ Nicolet, W. E., "Advanced Methods for Calculating Radiation Transport in Ablation Product Contaminated Boundary Layers," NASA CR-1656, Sept. 1970.

${ }^{19}$ Nicolet, W. E., "User's Manual for the Generalized Radiation Transfer Code (RAD/EQUIL)," NACA CR-116353, Oct. 1969.

${ }^{20}$ Shinn, J. L., "Optical Absorption of Carbon and Hydrocarbon Species from Shock Heated Acetylene and Methane in the 135-220 m Wavelength Range." Thermophysics of Atmospheric Entry, edited by T. E. Horton, Vol. 82, Progress in Astronautics and Aeronautics, AIAA, New York, 1982, pp. 68-80.

${ }^{21}$ Armaly, B. F., and Sutton, K., "Viscosity of Multicomponent Partially Ionized Gas Mixtures," Aerothermodynamics and Planetary Entry, edited by A. L. Crosbie, Vol. 77, Progress in Astronautics and Aeronautics, AIAA, New York, 1982, pp. 335-350.
${ }^{22}$ Mason, E. A., and Saxena, S. C., "Approximation Formula for the Thermal Conductivity of Gas Mixtures," Physics of Fluids, Vol. 1, No. 5, 1958, pp. 361-369.

${ }^{23}$ Cebeci, T., and Smith, A. M. O., Analysis of Turbulent Boundary Layers, Academic, New York, 1974, pp. 211-257.

${ }^{24}$ Gupta, R. N., "Turbulence Modeling of Flow fields with Massive Surface Ablation," Journal of Spacecraft and Rockets, Vol. 20, No. 6, 1983, pp. 531538.

${ }^{25}$ Anderson, E. C., Moss, J. N., and Sutton, K., "Turbulent Viscous-ShockLayer Solutions with Strong Vorticity Interaction," Journal of Spacecraft and Rockets, Vol. 14, No. 1, 1977, pp. 32-37.

${ }^{26}$ Park, C., "Radiation Enhancement by Nonequilibrium in Earth's Atmosphere," Journal of Spacecraft and Rockets, Vol. 22, No. 1, 1985, pp. 27-36.

T. C. Lin Associate Editor 

